\begin{tabular}{|c|c|}
\hline Title & $\begin{array}{l}\text { A symmetric spin relaxation induced by residual electron spin in semiconductor quantum-dot-superlattice hybrid } \\
\text { nanosystem }\end{array}$ \\
\hline Author(s) & Hiura, Satoshi; Hatakey ama, Saeko; Takay ama, Junichi; Muray ama, A kihiro \\
\hline Citation & $\begin{array}{l}\text { A pplied phy sics letters, } 116(26), 262407 \\
\text { https://doi org/10.1063/5.0010754 }\end{array}$ \\
\hline Issue Date & $2020-06-29$ \\
\hline Doc URL & http:/hdl.handle.net/2115/82072 \\
\hline Rights & $\begin{array}{l}\text { This article may be downloaded for personal use only. Any other use requires prior permission of the author and A IP } \\
\text { Publishing. This article appeared in A ppl. Phys. Lett. 116(26), } 262407 \text { (2020) and may be found at } \\
\text { http://doi.org/10.1063/5.0010754. }\end{array}$ \\
\hline Type & article \\
\hline File Information & 5.0010754.pdf \\
\hline
\end{tabular}

Instructions for use 


\section{Asymmetric spin relaxation induced by residual electron spin in semiconductor quantum-dot-superlattice hybrid nanosystem}

Cite as: Appl. Phys. Lett. 116, 262407 (2020); https://doi.org/10.1063/5.0010754

Submitted: 16 April 2020 . Accepted: 19 June 2020 . Published Online: 01 July 2020

Satoshi Hiura (D), Saeko Hatakeyama, Junichi Takayama (D), and Akihiro Murayama (D)
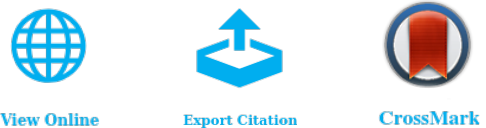

View Online

Export Citation

\section{ARTICLES YOU MAY BE INTERESTED IN}

A hybrid structure light-emitting device based on a $\mathrm{CsPbBr}_{3}$ nanoplate and two-dimensional materials

Applied Physics Letters 116, 263103 (2020); https://doi.org/10.1063/5.0014497

Stabilization of exponential number of discrete remanent states with localized spin-orbit torques

Applied Physics Letters 116, 262405 (2020); https://doi.org/10.1063/5.0005964

Defect properties of $\mathrm{Sb}_{2} \mathrm{Se}_{3}$ thin film solar cells and bulk crystals

Applied Physics Letters 116, 261101 (2020); https://doi.org/10.1063/5.0012697

\section{Lock-in Amplifiers up to $600 \mathrm{MHz}$}
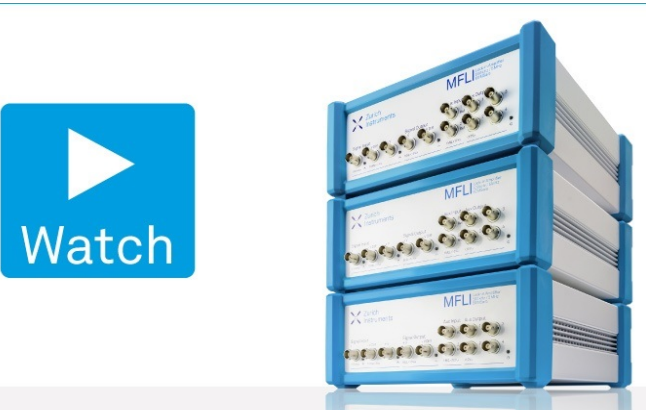


\title{
Asymmetric spin relaxation induced by residual electron spin in semiconductor quantum-dot-superlattice hybrid nanosystem
}

\author{
Cite as: Appl. Phys. Lett. 116, 262407 (2020); doi: 10.1063/5.0010754 \\ Submitted: 16 April 2020 - Accepted: 19 June 2020 • \\ Published Online: 1 July 2020
}

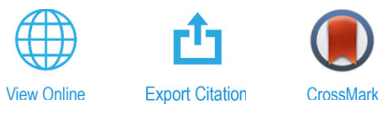

Satoshi Hiura, ${ }^{\text {a) }}$ (D Saeko Hatakeyama, Junichi Takayama, (D) and Akihiro Murayama (D)

\author{
AFFILIATIONS \\ Faculty of Information Science and Technology, Hokkaido University, Kita 14, Nishi 9, Kita-ku, Sapporo 060-0814, Japan
}

a) Author to whom correspondence should be addressed: hiura@ist.hokudai.ac.jp

\begin{abstract}
Asymmetric spin relaxation induced by the residual electron spin in semiconductor quantum dots (QDs) adjacent to a superlattice (SL) was studied using spin- and time-resolved photoluminescence under the selective photoexcitation of the SL miniband states. Spin-polarized electrons were photoexcited in the SL barrier and then injected into the QDs through spin-conserving tunneling. The spin-polarized electron transport and the faster transport of the electrons as compared to the holes generate the residual majority electron spins in the QDs. A reversal of the optical spin polarity was observed at the ground state of the QDs, depending on the excitation powers. A rate equation analysis considering the individual spin-flip times between spin-split QD states indicates that the polarity reversal originates from the asymmetric spin-flip process at the excited state of the QDs. The asymmetric spin relaxation is associated with the selective relaxation of the spin-flipped electron and hole to the unoccupied ground state, which is induced by the existence of the residual majority electron spin at this state. In addition, we observed a clear recovery of the optical spin polarity by eliminating the existence of the residual electron spin through heavy p-doping. These findings are important to attain a fundamental understanding of the spin relaxation mechanism within the QDs and provide an insight into the manipulation of the optical spin polarity by controlling the residual electron spins in the QDs.
\end{abstract}

Published under license by AIP Publishing. https://doi.org/10.1063/5.0010754

To realize future semiconductor opto-spintronic devices such as a spin-polarized light emitting diode, ${ }^{1-3}$ the retention of the spin information is one of the most important elements. Semiconductor quantum dots (QDs) are the most promising material for the retention of the spin information owing to their three-dimensional quantum confinements. ${ }^{4-6}$ QDs also show superior luminescence properties owing to a quantum confinement and resulting strong overlap of the carrier wavefunctions. In addition to the retention of the spin information, spin transport in semiconductor barriers is also a key element to optospintronic applications because electron spins are rapidly depolarized owing to the D'yakonov and Perel, ${ }^{7}$ Bir et al., ${ }^{8}$ Elliott, ${ }^{9}$ and Yafet ${ }^{10}$ mechanisms and hyperfine interaction. ${ }^{11,12}$ To overcome this issue, electric field effects on spin transport properties in single and multiple GaAs/AlGaAs quantum wells (QWs), ${ }^{13,14}$ and spin transport properties in $\mathrm{Ge} / \mathrm{SiGe}$ multiple QWs ${ }^{15}$ have been investigated. Interestingly, quantum spin transport from a semiconductor superlattice (SL) to QDs has recently been demonstrated, where the spin state can be fully conserved in the penetration of its wavefunction. ${ }^{16}$ In this case, the tunneling rate of electrons should be faster than that of holes owing to the lower effective mass. The faster transport of electrons causes an electron-rich situation inside the QDs, i.e., the generation of residual electrons at the QD emissive states. Such excess electrons in the QDs induce a luminescence counter-polarized to the initial excitation owing to the electron-hole spin flip-flop interactions. ${ }^{17-25}$ Although the optical spin properties in QDs with excess electrons have been studied using n-doped QDs ${ }^{17-22}$ or by means of an applied electric bias used to control the charge state of the QDs, ${ }^{23-25}$ these properties have not been sufficiently explored in hybrid nanosystems. This understanding has significant implications for manipulating the optical spin properties of QD-based nanosystems.

In this study, a single layer of self-assembled $\mathrm{In}_{0.5} \mathrm{Ga}_{0.5} \mathrm{As}$ QDs with a $\mathrm{GaAs} / \mathrm{Al}_{0.15} \mathrm{Ga}_{0.85} \mathrm{As} \mathrm{SL}$ barrier laid on top was grown on the GaAs(100) substrate through molecular beam epitaxy. The schematic of the sample structure is shown in the inset in Fig. 1(a). The SL consists of a repeat of GaAs QW $(10 \mathrm{~nm}) / \mathrm{Al}_{0.15} \mathrm{Ga}_{0.85} \mathrm{As}$ barrier $(5 \mathrm{~nm})$. Details of the sample growth and the QD structures are described in the supplementary material. Circularly polarized photoluminescence (PL) and its time-resolved experiments were conducted under 

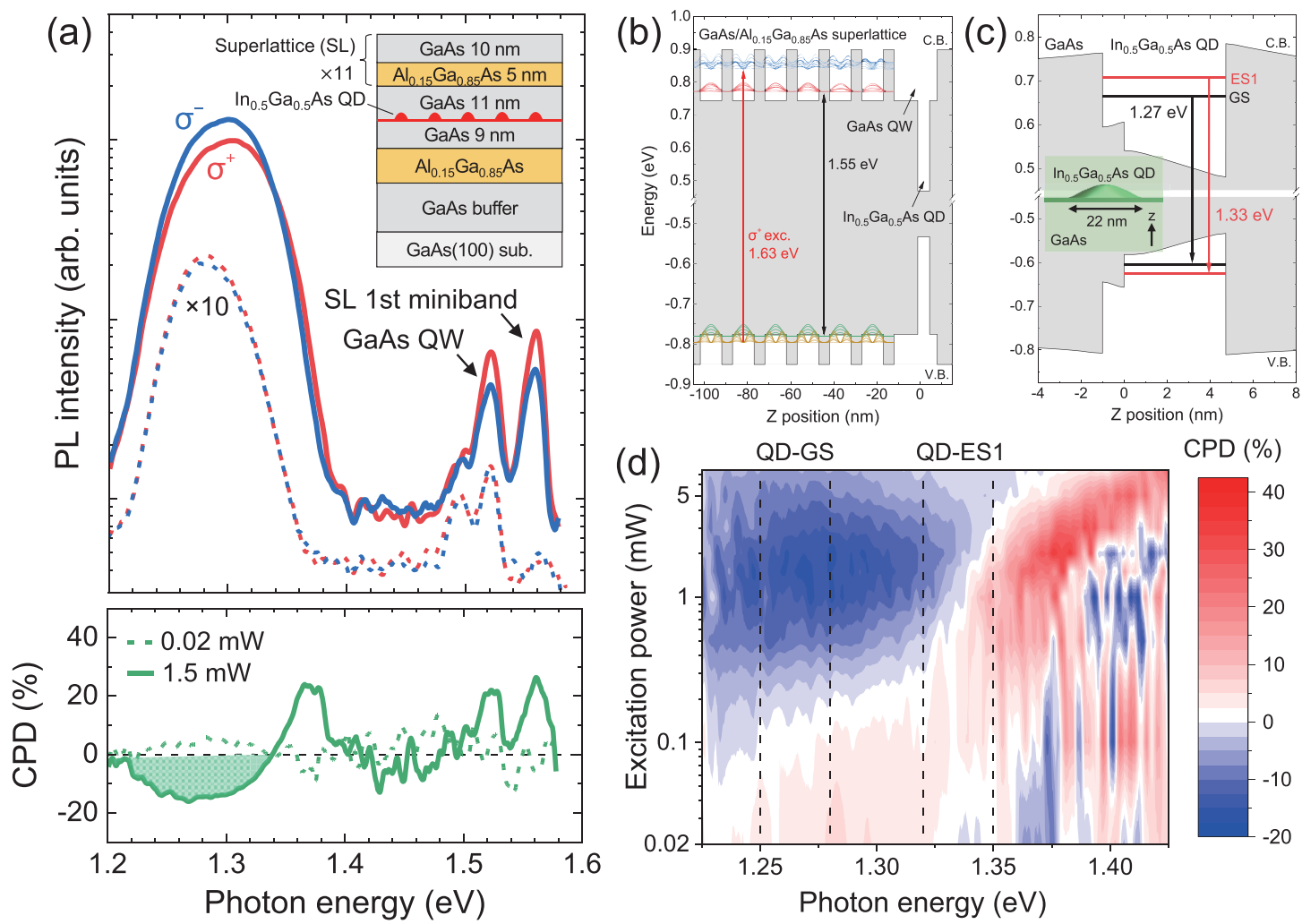

FIG. 1. (a) Circularly polarized time-integrated PL spectra and corresponding CPD at $1.5 \mathrm{~mW}$. The corresponding results at $0.02 \mathrm{~mW}$ are also shown by the dotted lines. The inset shows a schematic of the sample structure. (b) One-dimensional calculation result of electron and hole wavefunctions in the first and second miniband states for GaAs/ $\mathrm{Al}_{0.15} \mathrm{Ga}_{0.85} \mathrm{As} \mathrm{SL}$. (c) Three-dimensional calculation results of the band profiles and the eigenstates for an $\mathrm{In}_{0.5} \mathrm{Ga}_{0.5} \mathrm{As} \mathrm{QD}$ with a base length of $22 \mathrm{~nm}$ along the stacking direction. (d) Contour map of CPD values as functions of the photon energy and excitation power.

$\sigma^{+}$-polarized excitation at $6 \mathrm{~K}$. The excitation energy was tuned to $1.63 \mathrm{eV}$ to selectively excite the second miniband states in the SL as shown in Fig. 1(b). Here, the polarization of electron spins generated in the GaAs QW is expected to be $50 \%$ according to the optical selection rule when taking the valence-band mixing into account. ${ }^{26}$ The circular polarization degree (CPD), measured in QDs, is CPD $=\left(I_{\sigma+}-I_{\sigma-}\right) /\left(I_{\sigma+}+I_{\sigma-}\right)$, where $I_{\sigma \pm}$ denotes the $\sigma^{ \pm}$-polarized PL intensity, reflecting the polarization of electron spins at QD emissive states. ${ }^{16}$ Details of the optical characterization are described in the supplementary material. One- and three-dimensional quantum simulations were also conducted to determine the SL miniband states, the ground state (GS), and the first excited state (ES1) of QD using the commercial software package nextnano. ${ }^{27}$

Figure 1(a) shows the circularly polarized time-integrated PL spectra and corresponding CPD measured at an excitation power of $1.5 \mathrm{~mW}$, where four PL peaks can be observed. First, we attribute the sharp PL at $1.55 \mathrm{eV}$ to the first miniband states. This value coincides with the calculated value shown in Fig. 1(b), which demonstrates that the SL was grown as designed. Another sharp PL at $1.52 \mathrm{eV}$ can be attributed to the GaAs QW surrounding the QD. The small peak at $1.49 \mathrm{eV}$ is related to the band-to-acceptor transition associated with the residual carbon impurities in the GaAs. ${ }^{28}$ The broad PL emission at $1.20-1.40 \mathrm{eV}$ comes from the QD-GS and QD-ES. The PL spectra measured at $0.02 \mathrm{~mW}$ revealed a QD-GS position of $1.28 \mathrm{eV}$. This value is also in good agreement with that obtained from a threedimensional calculation. Figure 1 (c) shows the calculated band profiles and eigenstates for an $\operatorname{In}_{0.5} \mathrm{Ga}_{0.5} \mathrm{As}$ QD with a base length of $22 \mathrm{~nm}$ along the stacking direction, where the strain distribution was considered. The base length was determined based on a structural analysis of the reference QDs (Fig. S1). This calculation shows a QD-GS of $1.27 \mathrm{eV}$ and a QD-ES1 of $1.33 \mathrm{eV}$.

Next, we discuss the excitation power dependence of the PL-CPD properties. As shown in Fig. 1(a), a positive CPD of $+6 \%$, corresponding to parallel polarization under the initial excitation, can be seen at a QD-GS at $0.02 \mathrm{~mW}$. By contrast, remarkable negative CPD values appear within a wide energy region including QD-GS and QD-ES1 at $1.5 \mathrm{~mW}$. The PL emission mainly originates from the electron spins anti-parallel to the initial excitation. Here, a high positive CPD of $+25 \%$ was observed at a higher QD-ES, which is closer to the SL. An initial CPD of $50 \%$ was obtained at the QD-ES (Fig. S2), which demonstrates a spin-conserved electron transport from the SL to the QDs. ${ }^{16}$ These results indicate that the reversal of the optical spin polarity was caused by the characteristic spin relaxation mechanism within the QDs.

Figure 1(d) shows a contour map of the CPD values as functions of the photon energy and excitation power. Here, the energy ranges of QD-GS and QD-ES1 are defined as $1.25-1.28$ and $1.32-1.35 \mathrm{eV}$, respectively, based on the calculation results described above. The negative CPD of the QD-GS reached a maximum of $-15 \%$ at $1.5 \mathrm{~mW}$ 

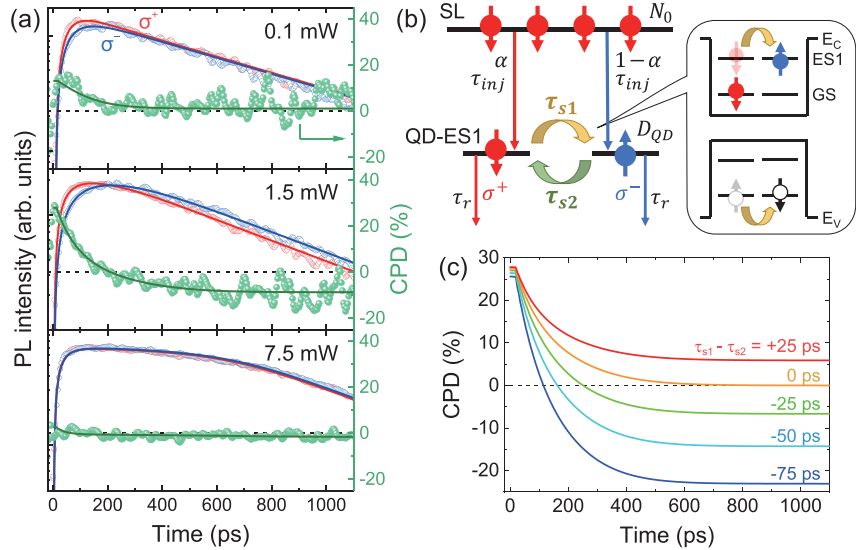

FIG. 2. (a) Circularly polarized PL time profiles and corresponding CPD of the QDES1 measured at $0.1,1.5$, and $7.5 \mathrm{~mW}$. The solid lines indicate the best-fitted results of the rate equation. (b) Schematic model of spin-split rate equation, where the individual spin-relaxation times, $\tau_{s 1}$ and $\tau_{s 2}$, are considered. (c) The simulation results of the transient CPD behavior as a function of time by varying $\tau_{s 1}$ with a fixed value of $\tau_{\mathrm{s} 2}=200 \mathrm{ps}$.

and then approached $0 \%$ with a further increase in the excitation power. Such negative CPD properties of QD-PL were previously explained by a spin flip-flop transition through scattering at QD-ES1 based on the formation of a negatively charged exciton with excess electrons in the QDs. ${ }^{17-25}$ In this study, the transfer of electrons should be faster than that of holes owing to the faster tunneling rate within the SL. Therefore, excess electrons in the QDs can occur as with ndoped QDs. ${ }^{17-22}$

To reveal the excitation power dependence of PL-CPD properties, we performed a time-resolved analysis of PL and its CPD of QD-ES1. Figure 2(a) shows the circularly polarized PL time profiles and corresponding CPD measured at 0.1, 1.5, and $7.5 \mathrm{~mW}$. At $0.1 \mathrm{~mW}$, an initial positive $\mathrm{CPD}$ of $+15 \%$ appeared and then gradually decreased to $0 \%$ within 300 ps. At $1.5 \mathrm{~mW}$, the CPD value steeply decreased from $+30 \%$ initially to $0 \%$ at $200 \mathrm{ps}$, followed by a slow evolution of the negative $\mathrm{CPD}$ down to $-10 \%$ during the entire radiative lifetime. At 7.5 $\mathrm{mW}$, the CPD was almost $0 \%$ regardless of time. To quantitatively understand these CPD behaviors, we performed a rate equation analysis. The rate-equation model is schematically illustrated in Fig. 2(b). Here, we assume the spin injection from the SL to the QD-ES1, where a spin flip-flop transition through scattering can occur. ${ }^{17-25}$ The rate equations can be written as follows:

$$
\begin{gathered}
\frac{d N_{0}}{d t}=\frac{-N_{o}}{\tau_{i n j}}\left\{\alpha\left(1-\frac{N_{Q D}^{\sigma+}}{D_{Q D}}\right)+(1-\alpha)\left(1-\frac{N_{Q D}^{\sigma-}}{D_{Q D}}\right)\right\}, \\
\frac{d N_{Q D}^{\sigma+}}{d t}=\frac{N_{o}}{\tau_{i n j}} \alpha\left(1-\frac{N_{Q D}^{\sigma+}}{D_{Q D}}\right)-\frac{N_{Q D}^{\sigma+}}{\tau_{r}}-\frac{N_{Q D}^{\sigma+}}{\tau_{s 1}}+\frac{N_{Q D}^{\sigma-}}{\tau_{s 2}}, \\
\frac{d N_{Q D}^{\sigma-}}{d t}=\frac{N_{o}}{\tau_{i n j}}(1-\alpha)\left(1-\frac{N_{Q D}^{\sigma-}}{D_{Q D}}\right)-\frac{N_{Q D}^{\sigma-}}{\tau_{r}}-\frac{N_{Q D}^{\sigma-}}{\tau_{s 2}}+\frac{N_{Q D}^{\sigma+}}{\tau_{s 1}},
\end{gathered}
$$

where $N_{0}$ is the initial number of excitons with $\sigma^{+}$-polarization. Then, spin-conserved $\alpha N_{0}$ are injected from the SL into the spin-polarized
QD state, while the anti-polarized ones $(1-\alpha) N_{0}$ are injected into the opposite QD state because of spin relaxation during the injection. Therefore, the parameter of $\alpha$ indicates the fraction of spin conservation in the spin-injection process, where $\alpha=0.5$ corresponds to the full spin relaxation during the injection while $\alpha=0.75$ corresponds to the full spin conservation because the initial polarization of the photoexcited electron spins is $50 \%$ as described above. $\tau_{i n j}$ and $\tau_{r}$ are the time constants for the spin injection from the SL to the QD states and for the energy relaxation from these QD states including radiative and nonradiative decaying processes, respectively. Moreover, $D_{Q D}$ denotes the density of states for the QDs and $N_{Q D}^{\sigma \pm}$ denotes the number of excitons in the QD state with $\sigma^{ \pm}$-polarization. Here, the individual time constants of the spin relaxation between the spin-split QD states, $\tau_{s 1}$ and $\tau_{s 2}$, are introduced, which were not considered in the previous studies. Note that $\tau_{s 1(2)}$ includes both the inherent spin relaxation process and the characteristic spin-flip process associated with the electron-hole spin flip-flop interactions [Fig. 2(b)]. Because the spinflip time should be much faster than the inherent spin-relaxation time of about $1 \mathrm{~ns},{ }^{29,30} \tau_{s 1(2)}$ strongly depends on the spin-flip time. In fact, a drastic reversal of the CPD polarity with decay times of $10-100$ ps was reported. ${ }^{17,23,24}$ Here, the spin-flip time between the spin-split QD states may be different because the spin flip-flop interactions are linked to the occupation of QD-ES1. ${ }^{22}$

Simulations of the transient CPD behavior as a function of time by varying $\tau_{s 1}$ are shown in Fig. 2(c). Here, $\tau_{s 1}$ was varied from 125 to $225 \mathrm{ps}$ at a fixed value of $\tau_{s 2}=200 \mathrm{ps}$. No substantial dependence of the transient CPD behavior on $\tau_{s 2}$ was obtained. The parameter values of $\tau_{i n j}$ and $\tau_{r}$ are set as 55 and 500 ps, respectively, obtained from the conventional rate-equation analysis of the data at $1.5 \mathrm{~mW}$ (Fig. S3), where a clear negative CPD appeared. The parameter $\alpha=0.65$ was also determined from the same data, which exhibits the initial CPD value of $\sim 30 \%$. As $\tau_{s 1}$ becomes faster than $\tau_{s 2}$, the CPD decay becomes steeper; thus, the maximum negative CPD becomes larger. This simulation suggests that the negative CPD property originates from the asymmetric spin-flip process between the spin-split QD states. The least-square fittings of the rate equations for the circularly polarized time-resolved PL and its CPD are shown in Fig. 2(a) (see the solid lines). The slow evolution of the negative CPD as well as the circularly polarized transient PL can be fully expressed. A combination of two fitting parameters, $\tau_{s 1}$ and $\tau_{s 2}$, can explain all PL time profiles and the resulting CPD behavior.

Figure 3(a) shows the averaged CPD of the QD-GS and the difference in the spin-flip time $\left(\tau_{s 1}-\tau_{s 2}\right)$ deduced from the above rateequation fitting as a function of the excitation power. Here, the averaged CPD of the QD-GS is defined as a simple average value of the data points between $1.25 \mathrm{eV}$ and $1.28 \mathrm{eV}$ in the CPD spectrum. A clear correlation between these behaviors can be seen. The maximum negative CPD appears when the difference in spin-flip time is the largest. The excitation power dependence of these behaviors can be explained by considering the degree of occupation of QD-GS and QD-ES1, as illustrated in Fig. 3(b). At low excitation powers, the spins generated in the SL close to the QD or in the QW surrounding the QD can mainly contribute to the QD-PL emission, as described in Fig. S4. In this case, in addition to the small amount of spin injection into the QDs, the difference in the number of electrons and holes injected is relatively small, leading to no residual electron spins. The transferred spins are mainly injected into the QD-GS via a rapid relaxation from the 
(a)

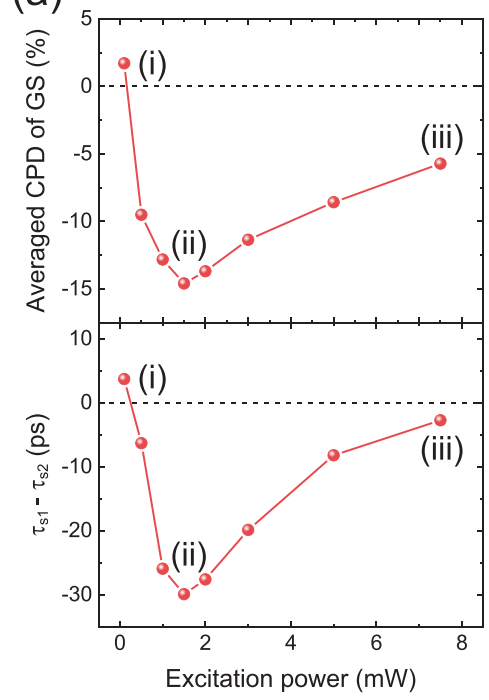

(b)

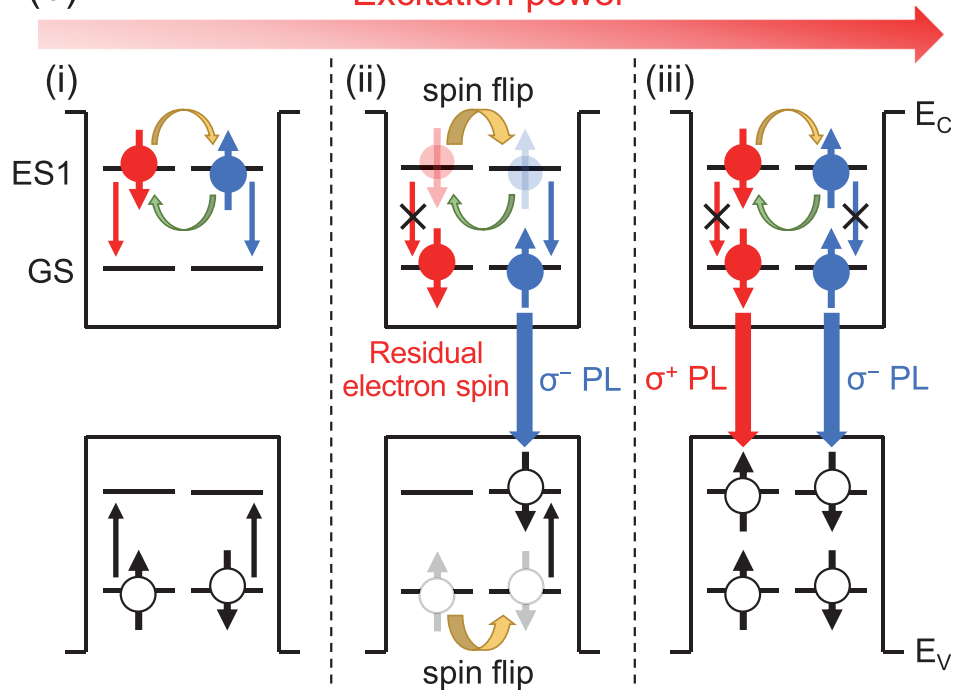

FIG. 3. (a) Averaged CPD of QD-GS and difference in spin-flip time $\left(\tau_{s 1}-\tau_{s 2}\right)$, deduced from the rate equation fitting as a function of the excitation power. (b) Schematic model describing the excitation power dependence of the optical spin polarity of the QDs.

QD-ES1. As the occupation of the QD-ES1 is sufficiently small, there is no difference in the spin-flip time. As a result, a positive CPD corresponding to parallel polarization under the initial excitation is observed. By contrast, at moderate excitation powers, the injection of the spins generated in the SL far from the QD increases, as described in Fig. S4. In this case, since the transport of electrons in the SL is faster than that of holes due to the lower effective mass, the number of electrons injected into the QDs should be larger. As a result, the number of electrons and holes in the QDs becomes unbalanced; thus, residual electrons appear at the QD-GS. Since the polarization of electron spins is retained during the transport, the spin direction of the residual electron is parallel to the initial excitation. Then, the parallel electron spins injected into the QD-ES1 cannot be directly relaxed to the QD-GS by the Pauli blocking. However, the combined energy relaxation of the electron and hole with simultaneous flips of their spins is allowed in the presence of anisotropic component of the exchange coupling. ${ }^{23}$ This spin-selective energy relaxation of the spin-flipped electron and hole can effectively reduce the possibility of the spin-upto-down-flip for electrons at the QD-ES1 [Fig. 3(b)], i.e., a faster $\tau_{s 1}$ than $\tau_{s 2}$. Here, the residual electron spins cannot emit light without the existence of hole spins that are radiative recombination pairs, while the spin-flipped electron and hole can radiatively recombine at the QD-GS, emitting negative CPD of PL. At high excitation powers, QDES1 as well as the QD-GS is fully occupied by both electron spins, i.e., the spin injection into the QD-ES1 increases. This state-filling effect prevents the energy relaxation from the QD-ES1 to the QD-GS as well as the spin flip-flop interaction at QD-ES1. As a result, the asymmetric spin-flip process disappears; thus, the CPD of QD-GS approaches 0\% owing to the state-filling effect in the QDs. ${ }^{29,30}$

We also investigate the effect of $\mathrm{p}$-doping on the PL-CPD properties. A structure similar to this study, with Be doping in the GaAs capping layer for embedding the QDs, was grown. The nominal doping concentration was $3 \times 10^{17} \mathrm{~cm}^{-3}$, corresponding to $\sim 23$ holes per QD based on the areal QD density. Figure 4(a) shows a contour map of the CPD values as functions of the photon energy and excitation power. We observed the disappearance of negative CPD properties by p-doping. Figure 4 (b) shows the averaged CPD of the QD-GS as a function of the excitation power. At $0.1 \mathrm{~mW}$, a positive CPD of $+11 \%$, as compared to $2 \%$ for the undoped sample, appeared. The enhanced $\mathrm{CPD}$ originates from the suppression of the anisotropic electron-hole exchange interaction. ${ }^{31,32}$ With increasing excitation power, the CPD value approached $0 \%$ owing to the increasing state-filling effect in the QDs. It should be noted that no reversal of the optical spin polarity was observed. This result demonstrates that p-doping fully eliminates the existence of the residual electron spins at the QD-GS, leading to a luminescence co-polarized to the initial excitation, as illustrated in the inset in Fig. 4(b). P-doping also significantly enhances the positive CPD values of the QD-ES1, as discussed in detail in the supplementary material (Fig. S5).
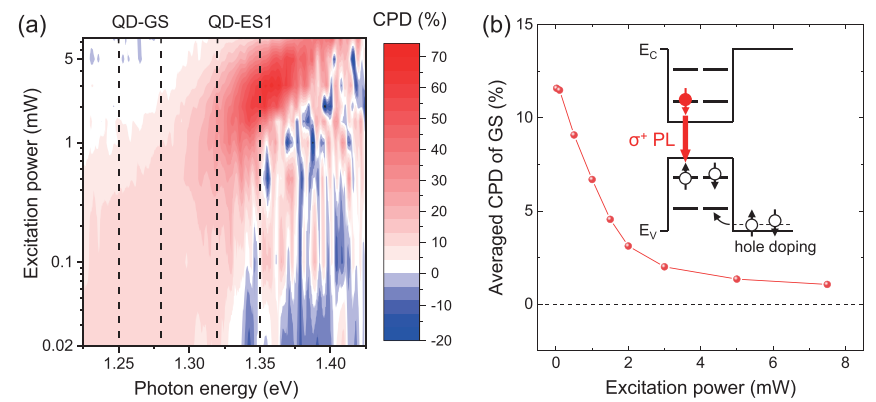

FIG. 4. (a) Contour map of CPD values as functions of photon energy and excitation power for $p$-doped sample. (b) Averaged CPD of QD-GS as a function of the excitation power. The inset shows a schematic to explain a co-polarized PL emission from QD-GS by p-doping. 
In conclusion, a spin relaxation process in the $\operatorname{In}_{0.5} \mathrm{Ga}_{0.5} \mathrm{As}$ QDs adjacent to the $\mathrm{GaAs} / \mathrm{Al}_{0.15} \mathrm{Ga}_{0.85} \mathrm{As}$ SL was studied using spin- and time-resolved PL with a selective photoexcitation of the SL miniband states. A clear reversal of the optical spin polarity appeared at the QDGS, depending on the excitation powers. A rate equation analysis considering the individual spin-relaxation times between spin-split QD states revealed that the asymmetric spin-flip process at the QD-ES1, associated with the residual electron spin at the QD-GS, is the main origin of the polarity reversal. We also observed a recovery of the optical spin polarity by p-doping. These results indicate that the optical spin property of the QDs strongly depends on the existence of the residual electron spins. Therefore, these findings provide an insight into a manipulation of the optical spin polarity by controlling the residual electron spins in the QDs.

See the supplementary material for sample growth conditions, QD structures, methods of optical characterization, initial CPD measured at a QD-ES higher than QD-ES1, parameter values of $\tau_{i n j}$ and $\tau_{r}$ in the rate-equation simulation, and excitation power dependences of the spin injection time and of the averaged CPD of the QD-ES1.

This work was supported by the Japan Society for the Promotion of Science (JSPS) under Grant Nos. 16H06359, 19K15380, and $19 \mathrm{H} 05507$.

\section{DATA AVAILABILITY}

The data that support the findings of this study are available from the corresponding author upon reasonable request.

\section{REFERENCES}

${ }^{1}$ Y. Chye, M. E. White, E. Johnston-Halperin, B. D. Gerardot, D. D. Awschalom, and P. M. Petroff, Phys. Rev. B 66, 201301 (2002).

${ }^{2}$ C. H. Li, G. Kioseoglou, O. M. J. van't Erve, M. E. Ware, D. Gammon, R. M. Stroud, B. T. Jonker, R. Mallory, M. Yasar, and A. Petrou, Appl. Phys. Lett. 86, 132503 (2005).

${ }^{3}$ L. Lombez, P. Renucci, P. F. Braun, H. Carrère, X. Marie, T. Amand, B. Urbaszek, J. L. Gauffier, P. Gallo, T. Camps, A. Arnoult, C. Fontaine, C. Deranlot, R. Mattana, H. Jaffrès, J.-M. George, and P. H. Binh, Appl. Phys. Lett. 90, 081111 (2007).

${ }^{4}$ D. Gammon, E. S. Snow, B. V. Shanabrook, D. S. Katzer, and D. Park, Science 273, 87 (1996).

${ }^{5}$ H. Gotoh, H. Ando, H. Kamada, A. Chavez-Pirson, and J. Temmyo, Appl. Phys. Lett. 72, 1341 (1998).

${ }^{6}$ M. Paillard, X. Marie, P. Renucci, T. Amand, A. Jbeli, and J. M. Gérard, Phys. Rev. Lett. 86, 1634 (2001).
${ }^{7}$ M. I. D’yakonov and V. I. Perel, Zh. Eksp. Teor. Fiz. 65, 362 (1973) , [Sov. Phys. JETP 38, 177 (1974)].

${ }^{8}$ G. L. Bir, A. G. Aronov, and G. E. Pikus, Zh. Eksp. Teor. Fiz. 69, 1382 (1975), [Sov. Phys. JETP 42, 705 (1976)].

${ }^{9}$ R. J. Elliott, Phys. Rev. 96, 266 (1954).

${ }^{10}$ Y. Yafet, Solid State Phys. 14, 1 (1963)

${ }^{11}$ G. Fishman and G. Lampel, Phys. Rev. B 16, 820 (1977).

${ }^{12}$ D. Paget, Phys. Rev. B 24, 3776 (1981).

${ }^{13}$ A. Hernández-Mínguez, K. Biermann, R. Hey, and P. V. Santos, Phys. Rev. B 94, 125311 (2016).

${ }^{14}$ G. Wang, B. L. Liu, A. Balocchi, P. Renucci, C. R. Zhu, T. Amand, C. Fontaine, and X. Marie, Nat. Commun. 4, 2372 (2013).

${ }^{15}$ C. Zucchetti, A. Ballabio, D. Chrastina, S. Cecchi, M. Finazzi, M. Virgilio, G. Isella, and F. Bottegoni, Phys. Rev. B 101, 115408 (2020).

${ }^{16}$ S. Hiura, K. Itabashi, K. Takeishi, J. Takayama, T. Kiba, and A. Murayama, Appl. Phys. Lett. 114, 072406 (2019).

${ }^{17}$ S. Cortez, O. Krebs, S. Laurent, M. Senes, X. Marie, P. Voisin, R. Ferreira, G. Bastard, J.-M. Gérard, and T. Amand, Phys. Rev. Lett. 89, 207401 (2002).

${ }^{18}$ S. Laurent, O. Krebs, S. Cortez, M. Senes, X. Marie, T. Amand, P. Voisin, and J. M. Gérard, Physica E 20, 404 (2004).

${ }^{19}$ V. K. Kalevich, I. A. Merkulov, A. Y. Shiryaev, K. V. Kavokin, M. Ikezawa, T. Okuno, P. N. Brunkov, A. E. Zhukov, V. M. Ustinov, and Y. Masumoto, Phys. Rev. B 72, 045325 (2005).

${ }^{20}$ S. Laurent, M. Senes, O. Krebs, V. K. Kalevich, B. Urbaszek, X. Marie, T. Amand, and P. Voisin, Phys. Rev. B 73, 235302 (2006).

${ }^{21}$ R. V. Cherbunin, S. Y. Verbin, T. Auer, D. R. Yakovlev, D. Reuter, A. D. Wieck, I. Y. Gerlovin, I. V. Ignatiev, D. V. Vishnevsky, and M. Bayer, Phys. Rev. B 80, 035326 (2009).

${ }^{22}$ M. W. Taylor, P. Spencer, and R. Murray, Appl. Phys. Lett. 106, 122404 (2015).

${ }^{23}$ M. Ikezawa, B. Pal, Y. Masumoto, I. V. Ignatiev, S. Y. Verbin, and I. Y. Gerlovin, Phys. Rev. B 72, 153302 (2005).

${ }^{24}$ B. Pal, S. Y. Verbin, I. V. Ignatiev, M. Ikezawa, and Y. Masumoto, Phys. Rev. B 75, 125322 (2007).

${ }^{25}$ H. Chen, S. Hiura, J. Takayama, S. Park, K. Sueoka, and A. Murayama, Appl. Phys. Lett. 114, 133101 (2019).

${ }^{26}$ F. Meier and B. Zakharchenya, Optical Orientation (Elsevier, North Holland, Amsterdam, 1984).

${ }^{27}$ S. Birner, T. Zibold, T. Andlauer, T. Kubis, M. Sabathil, A. Trellakis, and P. Vogl, IEEE Trans. Electron Devices 54, 2137 (2007).

${ }^{28}$ V. Swaminathan, D. L. Van Haren, J. L. Zilko, P. Y. Lu, and N. E. Schumaker, J. Appl. Phys. 57, 5349 (1985).

${ }^{29}$ T. Kiba, X. Yang, T. Yamamura, Y. Kuno, A. Subagyo, K. Sueoka, and A. Murayama, Appl. Phys. Lett. 103, 082405 (2013).

${ }^{30}$ T. Yamamura, T. Kiba, X. Yang, J. Takayama, A. Subagyo, K. Sueoka, and A. Murayama, J. Appl. Phys. 116, 094309 (2014).

${ }^{31}$ A. I. Tartakovskii, J. Cahill, M. N. Makhonin, D. M. Whittaker, J.-P. R. Wells, A. M. Fox, D. J. Mowbray, M. S. Skolnick, K. M. Groom, M. J. Steer, and M. Hopkinson, Phys. Rev. Lett. 93, 057401 (2004).

${ }^{32}$ P.-F. Braun, X. Marie, L. Lombez, B. Urbaszek, T. Amand, P. Renucci, V. K. Kalevich, K. V. Kavokin, O. Krebs, P. Voisin, and Y. Masumoto, Phys. Rev. Lett. 94, 116601 (2005). 